\title{
Comparative advantage for the areas irrigated with underground blue water in North China Plain
}

\author{
Lixi Zhao ${ }^{\mathrm{a}}$, Yunkai Li $i^{\mathrm{a}, \mathrm{b}, *}$, Fang Jianga, Hui Wanga ${ }^{\mathrm{a}}$, Shumei Renª, \\ Yaoze Liu and Zhiyun Ouyang ${ }^{\mathrm{b}}$ \\ ${ }^{a}$ Water Conservancy and Civil Engineering College, China Agricultural University, Beijing 100083, China \\ ${ }^{b}$ State Key Laboratory of Urban and Regional Ecology, Research Centre for Eco-Environmental Sciences, Chinese Academy of \\ Sciences, Beijing 100085, China \\ *Corresponding author. E-mail: liyunkai@126.com
}

\begin{abstract}
North China Plain is one of the most important grain-producing areas in China. Because of unevenly distributed precipitation in this semi-arid area, crop production largely relies on underground blue water (UBW) to irrigate, overexploitation of which causes a lot of environmental problems. In this paper, we first defined the representative division of winter wheat irrigation water production efficiency with a support vector machine and genetic algorithm coupling algorithm, then established a model for evaluating the comparative advantage of UBW production efficiency by combining the effective precipitation with the UBW depth, calculated its value in each city, and further proposed the reasonable irrigation water requirement and its distribution under different grain reserves at the targeting year. The result showed that when the typical precipitation $(P)$ is $25 \%$ in a targeting year, there is no need to irrigate with UBW; when $P=50 \%$, supplying $5 \%$ of total winter wheat production (TWP) could save $15 \%$ of UBW; when $P=75 \%$, supplying $5 \%$ of TWP could save $11 \%$ of UBW; and when $P=75 \%$, supplying $10 \%$ of TWP could save up to $28 \%$ of UBW.
\end{abstract}

Keywords: Comparative advantage; Irrigation water production efficiency; North China Plain; Regulation and management; Underground blue water

\section{Introduction}

North China Plain, located in the arid and semi-arid area, is one of the most important crop-producing areas in China. People use $18.3 \%$ of the area, which produces $22 \%$ of the crops in China. The precipitation in North China Plain is unevenly distributed. The annual average precipitation is $470-912 \mathrm{~mm}$, but about $65-80 \%$ of precipitation is from June to September (Gong, 2003). In addition, a lack of surface water makes it difficult to satisfy the requirement for irrigation water. To solve this problem, more groundwater could be exploited, but this would cause severe overexploitation and environmental problems (Liu, 2003).

doi: 10.2166/wp.2015.114

CC IWA Publishing 2015 
The concept of green water and blue water was raised in 1995 and the major blue water in North China Plain is groundwater. Thus, to make full use of green water and improve the efficiency of underground blue water (UBW), efficient regulation and management are important to solve groundwater overexploitation. Some scholars have evaluated the UBW production efficiency in some dry areas (Ferrara, 2007; Machiwal et al., 2011), and proved that it could be used to guide the management of groundwater resources in practice. In their studies, some scholars have used features such as photosynthetic available radiation, average accumulated temperature, and soil organic matter in crop-water production functions of different crops in different areas (Kipkorir et al., 2002; Liu et al., 2002). Although some studies focused on North China Plain (Luo et al., 2006), blue water and green water were not separated in crop-water production functions when calculated. It is important to pay more attention to the irrigation efficiency of UBW in order to solve issues like groundwater over-mining and vulnerable ecological environment protection. To this end, we first collected agricultural meteorological data and UBW depth of 21 cities in North China Plain, then obtained the winter wheat irrigation water production efficiency in representative divisions using a support vector machine and genetic algorithm (SVM-GA) coupling algorithm, and finally established a model to evaluate the comparative advantage of UBW production efficiency of each city by three features: winter wheat irrigation water production efficiency, the effective precipitation, and UBW depth. Based on the results, we put forward the reasonable irrigation water requirement and its distribution under different grain supply rates and typical targeting years.

\section{Materials and methods}

\subsection{Model for evaluating the comparative advantage of UBW production division}

Effective precipitation $(\eta)$, water production efficiency $(\theta)$, and the depth of UBW $(\rho)$ were used to construct a two-dimensional matrix of 21 cities in North China Plain based on the fuzzy classified theory ( $\mathrm{Li} \& \mathrm{Wu}, 1986)$ as shown in the following formula:

$$
\lambda=\left[\begin{array}{c}
\lambda_{1} \\
\lambda_{2} \\
\vdots \\
\lambda_{21}
\end{array}\right]=\left[\begin{array}{ccc}
\eta_{1} & \theta_{1} & \rho_{1} \\
\eta_{2} & \theta_{2} & \rho_{2} \\
\vdots & \vdots & \vdots \\
\eta_{21} & \theta_{21} & \rho_{21}
\end{array}\right]
$$

Effective precipitation is equal to the difference between total precipitation and actual evapotranspiration. After $\lambda$ is centralized and standardized, the fuzzy similar matrix was generated based on Euclidean distance to evaluate UBW production efficiency in North China Plain. The water production efficiency is calculated through

$$
\theta=\frac{f\left(\mathrm{ET}_{c \max }\right)}{\mathrm{ET}_{c \max }-\mathrm{ET}_{c \min }}
$$

where $f(x)$ is the crop-water production function, $\mathrm{ET}_{c \max }$ is the maximum value of the crop-water production function, and $\mathrm{ET}_{c \text { min }}$ is the minimum value of the irrigation efficiency function. The detailed explanation of these functions and coefficients will be given at the end of Section 2.3. 
The deeper the UBW $(\rho)$ is, the more severe the exploitation would be. The value of $\rho$ is used to evaluate groundwater exploitation

$\rho_{n}=1-\frac{h_{n}-h_{\min }}{h_{\max }-h_{\min }}$

where $h_{n}$ is the average depth of UBW in the certain region $n, h_{\max }$ is the maximum average depth of UBW in the certain region $n$, and $h_{\min }$ is the minimum average depth of UBW in the certain region $n$.

To calculate and rank the UBW production efficiency of winter wheat in different areas, $\eta, \theta$ and $\rho$ were regarded as the three coordinates in a three-dimensional coordinate system. A certain region was defined as a point in the three-dimensional coordinate system, and its distance from the origin point $\lambda_{n}$ could be expressed as

$\lambda_{n}=\sqrt{\eta_{n}^{2}+\theta_{n}^{2}+\rho_{n}^{2}}$

Through the equation, the comparative advantage for production efficiency of winter wheat irrigated with UBW could be obtained.

\subsection{Scenario analysis considering food security and UBW use quantity}

To establish a reasonable UBW distribution making utilization efficiency higher and ensuring food security, the following mathematical objective function was set up

$$
\begin{aligned}
& \min : \chi=\sum_{n=1}^{21} x_{n} \cdot s_{n} \\
& \text { s.t. } \mathrm{A} \leq \sum_{n=1}^{21} f_{n}\left(x_{n}\right) \times s_{n} \\
& W_{n r 2} \leq x_{n} \leq W_{n r 1}, \quad n=1,2, \ldots 21
\end{aligned}
$$

where $\chi$ is the total UBW quantity irrigated, $x_{n}$ is the UBW quantity irrigated in a certain region $n$; A is the quantity of winter wheat needed, $f_{n}\left(x_{n}\right)$ is crop-water production function in region $n, S_{n}$ is the area of winter wheat planted, and $W_{n r 1}$ and $W_{n r 2}$ are the upper and lower limits of irrigation water quantities, respectively, in a certain region $n$.

In the above equations, the weight of the comparative advantage of UBW irrigated in different places was considered equal. However, UBW distribution was different in every area, thus their weights were not the same. To solve the problem, the following optimization was carried out: (1) evaluating the comparative advantage of UBW production efficiency and classifying all areas into several irrigation groups; (2) arranging the higher utilization rate of irrigation groups to meet the demand of winter wheat; and (3) arranging the lower utilization rate of irrigation groups to meet the quantity demand for food security. 


\subsection{Method to determine the water production efficiency and its parameters' thresholds in representative divisions}

2.3.1. Method for water production efficiency of representative divisions. Light, heat, water, air, and nutrients are five basic elements of crop growth, and they have the same importance and nonsubstitutability (Duan et al., 2004). To obtain the regression coefficients of the crop-water production function in each region in North China Plain based on known observation sites, water production efficiency in a representative division was calculated based on the average photosynthetic available radiation, the average accumulated temperature and the soil organic matter using an SVM-GA coupling algorithm.

SVM was proposed by Vapnik and his colleagues in 1995 (Vapnik, 1995) as a new mechanical learning method. By choosing kernel functions, penalty terms and slack variables, the optimization problem was converted into a dual problem, and then a Lagrange function was used to obtain the corresponding classification function

$f(\mathbf{x})=\operatorname{sgn}\left[\sum_{j=1}^{N} \alpha_{j}^{*} y_{j} K\left(\mathbf{x}_{\mathbf{j}}, \mathbf{x}_{\mathbf{i}}\right)+b *\right]$

where $\mathbf{x}_{\mathbf{i}}$ is the training point, $y_{j}$ is the corresponding category label, $\alpha_{j}^{*}$ is the optimal solution, $K\left(\mathbf{x}, \mathbf{x}^{\prime}\right)$ is the kernel function, and $b^{*}$ is the optimal value of offset.

Different kernel functions have different effects on the generalization ability of SVM. Among the most common kernel functions, radial basis function (RBF), which has a good learning ability and fewer parameters (Lin \& Lin, 2003), has been widely used. It was chosen to classify winter wheat water production efficiency in this paper. And the genetic algorithm (GA), which was borrowed from the biological genetics point of view to obtain the optimal values (Koza, 1992), was used to search for the optimal solutions of the parameters of RBF, while cross inspection was used to search for the optimal threshold of parameters.

There were 17 experimental stations of winter wheat irrigation in North China Plain. These stations were classified into three categories in advance by regression coefficients of winter wheat in each site, resulting in six of them belonging to the first category, eight to the second category, and three to the third category. The steps are as follows.

First, data were collected from different departments: background information (including geographical location, latitude, longitude) came from the national irrigation experimental station nets; regression coefficients of the crop-water production function came from Irrigation Water Quota of Major Crops in North China (Duan et al., 2004); the spatial distribution grid figure of the average photosynthetically active radiation per year and the spatial distribution grid figure of the average accumulated temperature $\left(\geq 0{ }^{\circ} \mathrm{C}\right)$ per year came from the National Meteorological Information Center (NMIC); the spatial distribution grid figure of soil organic matter in North China Plain came from a 1:14000000 map of 'The Soil Organic Matter Map of China' by Data Center for Resources and Environmental Sciences of the Chinese Academy of Sciences.

Second, in the ArcGIS 10.0 interface, data of each irrigation observation site were extracted by using the spatial distribution grid figures collected and taken as training points. Appropriate kernel function and classifier were chosen to obtain the classification function $f(\mathbf{x})$. 
Finally, the data of each city were put into the model to obtain the appropriate water production efficiency of representative divisions.

2.3.2. Determining the parameters' threshold of water production efficiency. The relationship between water consumption and crop production could be described as the empirical crop-water production equation (Duan et al., 2004).

$y=a \mathrm{ET}_{c}^{2}+b \mathrm{ET}_{c}+c$

where $y$ is the production of crops, $\mathrm{ET}_{c}$ is the water consumption, and $a, b$, and $c$ are the regression coefficients of the function of winter wheat and water production.

The crop-water production quadratic function has an extreme value, the maximum crop production $\mathrm{ET}_{c \mathrm{max}}$.

$\mathrm{ET}_{c \max }=-\frac{b}{2 a}$

The coefficient of crop-water requirement is the quantity of water needed to produce $1 \mathrm{~kg}$ of crops in every hectare and the equation is

$K=\frac{\mathrm{ET}_{c}}{f\left(\mathrm{ET}_{c}\right)}$

It has a minimum

$\mathrm{ET}_{c \min }=\sqrt{\frac{c}{a}}$

The correlation of crop production and the coefficient of crop-water requirement with irrigated water increasing is shown in Figure 1. When function $K$ decreases, $y$ increases, and when $K$ decreases to $\mathrm{ET}_{c \mathrm{~min}}$, $y$ continues to increase; then $K$ and $y$ both increase, but when $y$ decreases from $\mathrm{ET}_{c \max }, K$ continues to increase. That means, if we continue to increase the UBW, the efficiency will decrease. In conclusion, this paper used an interval that is more economical (both $y$ and $K$ increase) to limit the quantity of irrigation water, that is,

$\mathrm{ET}_{\text {economic }} \in\left[\mathrm{ET}_{c \min }-P, \mathrm{ET}_{c \max }-P\right]$

where $P$ is effective precipitation.

\section{Results and analysis}

\subsection{Spatial distribution and representative division of water production efficiency in North China Plain}

The spatial distribution grid figures of average photosynthetically active radiation, soil organic matter and average accumulated temperature were obtained by using the spatial interpolation method, and then 


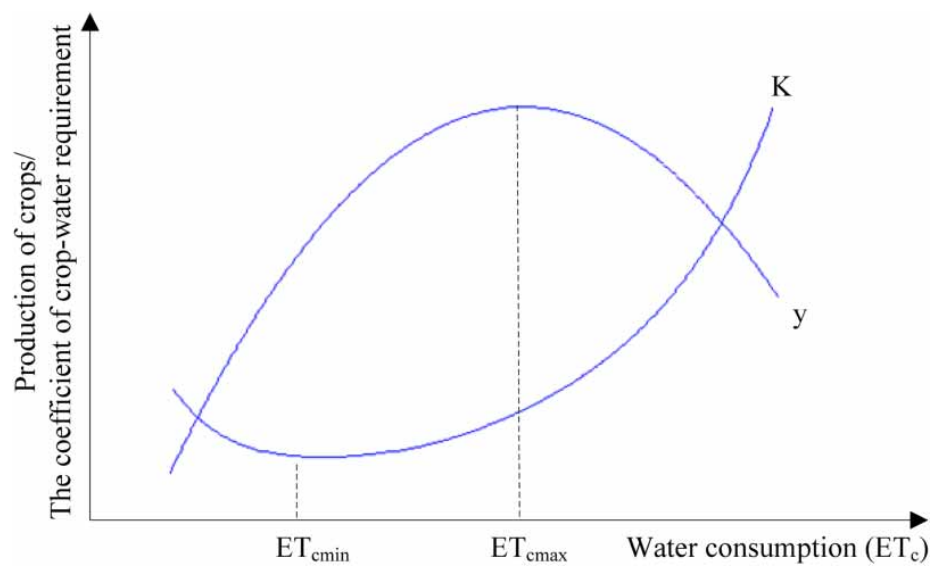

Fig. 1. The correlation of water consumption, crop production and the coefficient of crop-water requirement.

they were put into the SVM-GA model to classify water production efficiency in different regions (Figure 2). The result of the RBF kernel function with GA showed that the optimum penalty term, $C$, is equal to 2.47 and the optimum gamma is equal to 0.93 . Because the accuracy of classification was $95.5 \%$ by cross validation, SVM-GA had a good ability to classify the efficiency of irrigation water. Figure 2 shows that the water irrigation efficiency could be classified into three categories. The first category had the lowest irrigation water efficiency. Its representative areas included Beijing, Tianjin, Tangshan, Qinhuangdao, Langfang and Dongying in the north of North China Plain. Their average accumulated temperature $\left(\geq 0{ }^{\circ} \mathrm{C}\right)$ ranged from 1,700 to $2,000{ }^{\circ} \mathrm{C}$, which would affect the growth of winter wheat (Mao et al., 2005), while their soil organic matters were better than other categories, which could promote the growth of winter wheat (Wu et al., 2004). The second category had medium irrigation water efficiency. Its representative areas included Handan, Puyang, Liaocheng, Dezhou, Xingtai and Hengshui in the center of North China Plain. Their average photosynthetically active radiation was $1,520-1,570 \mathrm{MJ} / \mathrm{m}^{2}$, even $1,570-1,620 \mathrm{MJ} / \mathrm{m}^{2}$ in some places, which increases winter wheat production (Zeng et al., 1991). The third category had the highest irrigation water efficiency. Its representative regions included Anyang, Hebi, Xinxiang and Jiaozuo in the south of the plain. Their average accumulated temperature $\left(\geq 0{ }^{\circ} \mathrm{C}\right)$ ranged from 2,300 to $2,600{ }^{\circ} \mathrm{C}$, while their average photosynthetically active radiation was the lowest among the three categories.

\subsection{Comparative advantage and the spatial distribution of UBW irrigation production efficiency in North China Plain}

The effective precipitation during the growth period of winter wheat per year in North China Plain decreased from south to north in general by spatial interpolation (Figure 3). According to the depth chart of deep and shallow groundwater in 2005 in the Atlas for Groundwater in North China Plain, the whole groundwater distribution vector data graph was obtained with ArcGIS10.0 spatial data editing tools (Figure 4), showing that the depth of groundwater increases from the margins to the center and the maximum is over $80 \mathrm{~m}$.

From the extracted data, the comparative advantage of UBW was obtained using the fuzzy classified theory and calculation of the distance from origin. As shown in Figure 5, the comparative advantage of 


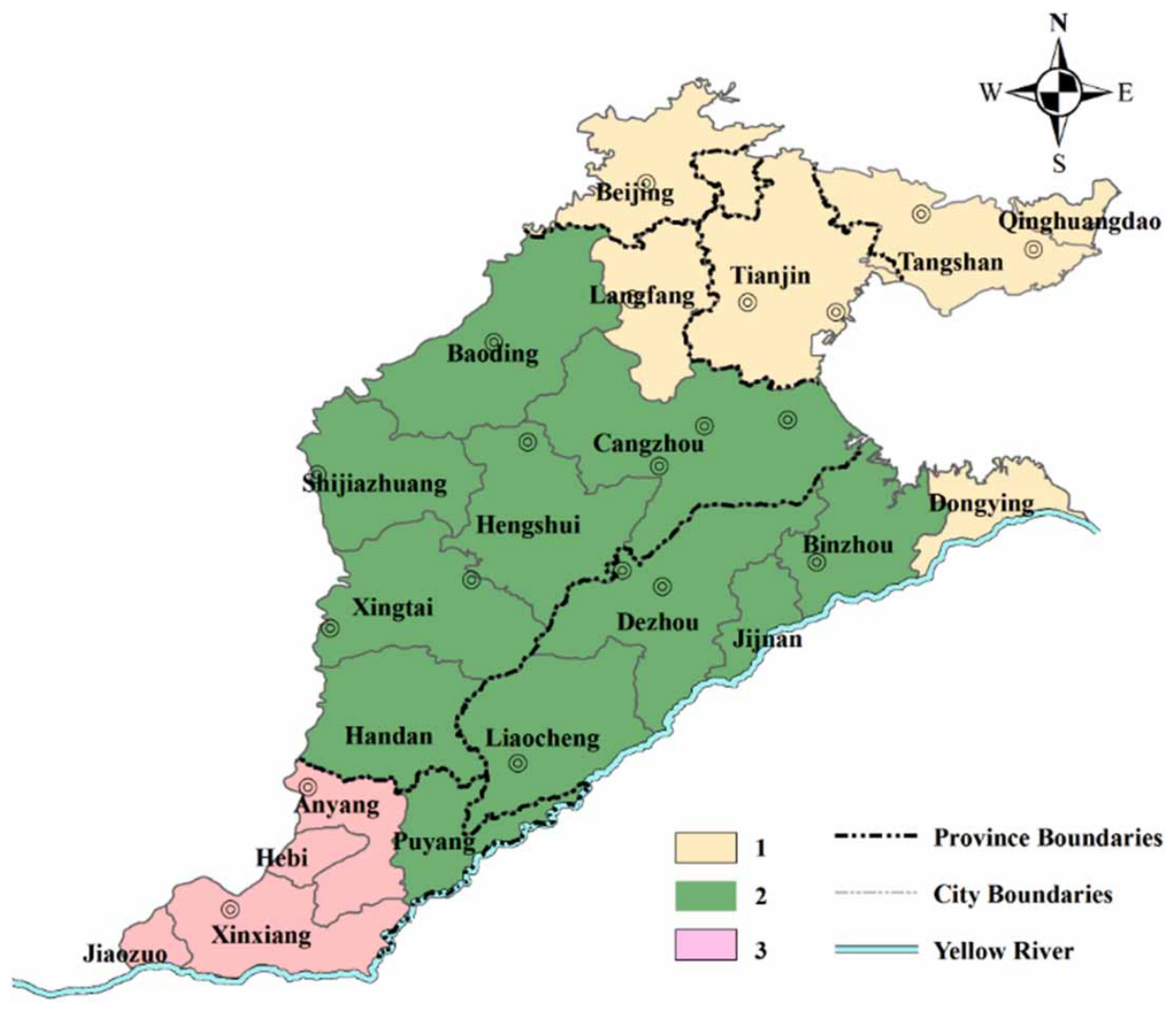

Fig. 2. Representative categories of water production efficiency.

UBW production efficiency decreased from the north and the south to the center, while it changed slightly from east to west. In detail, it was lower in the center than in other areas, and higher in the north than in the south. The average effective precipitation had significant influence on the efficiency of irrigation water. As shown in Figure 3, the fifth-category areas (Jiaozuo, Xinxiang, Hebi, Anyang and Puyang) in the south of the plain, which had precipitation of 180-240 mm, had the highest comparative advantage, while the first-category areas (Xingtai, Hengshui and Cangzhou) and the secondcategory areas (Dongying, Dezhou, Handan, Shijiazhuang and Baoding), which had precipitation of only 120-140 mm, had the lowest comparative advantage. Moreover, the depth of UBW could reflect the exploitation difficulty. As shown in Figure 4, the depth of UBW was less than $25 \mathrm{~m}$ in the fifth and fourth categories, meaning less exploitation; between 20 and $50 \mathrm{~m}$ in the third and second categories, and $50-70 \mathrm{~m}$ in the first category, indicating severe overexploitation.

\subsection{Rational management of $U B W$ use quantity at different effective precipitations in typical targeting years}

To manage and save the UBW, we proposed that the government could store or input some winter wheat when more UBW needed to be exploited. The winter wheat production in 2005 was used to 


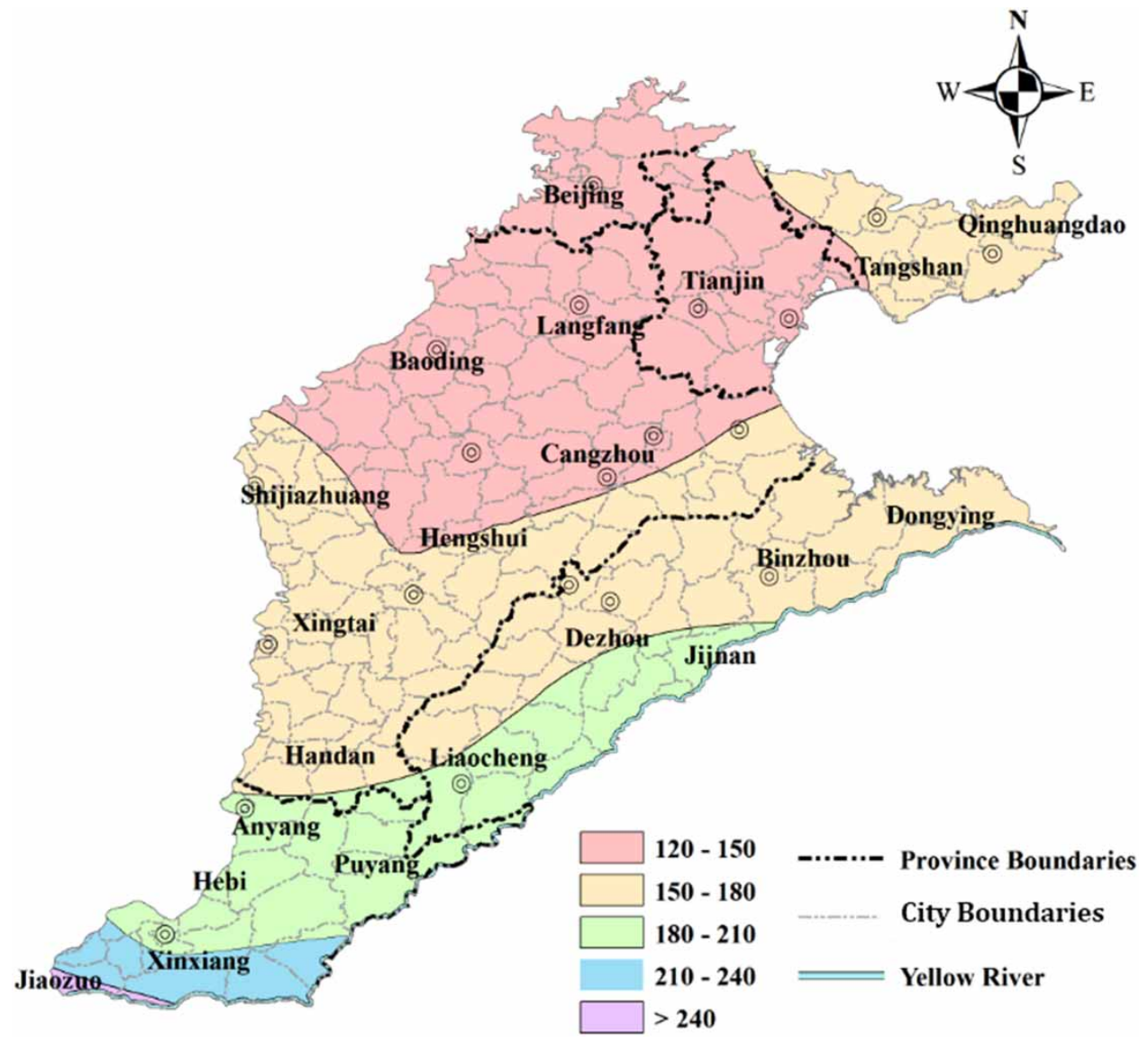

Fig. 3. Spatial distribution of effective precipitation of winter wheat per year.

predict the quantity of UBW needed under the conditions of three typical years. Assuming that when the effective precipitation $P=25 \%$, there is no need to supply UBW to the winter wheat reserve, because the precipitation is sufficient to ensure food security at this condition, the total quantity of irrigation water used in the year with $P=25 \%$ was considered as a threshold.

Figure 6(a) shows the result when there are no winter wheat needs to be supplied. When $P=25 \%$ in the targeting year, the UBW consumption is less, meaning that the consumption of UBW in firstcategory and second-category areas significantly decreased, while the third-category area needs to be fully irrigated to maintain the full capacity of crop production. When $P=50 \%$ in the targeting year, the UBW quantity exploited would be 1.15 -fold that of $P=25 \%$, in particular there is a comparative advantage of UBW in the second-category area in order to maintain the full capacity of crop production. When $P=75 \%$ in the targeting year, that is, there is a striking decrease in precipitation, the UBW quantity exploited would be 1.29 times that of $P=25 \%$ to maintain the full capacity to produce crop in all areas. 


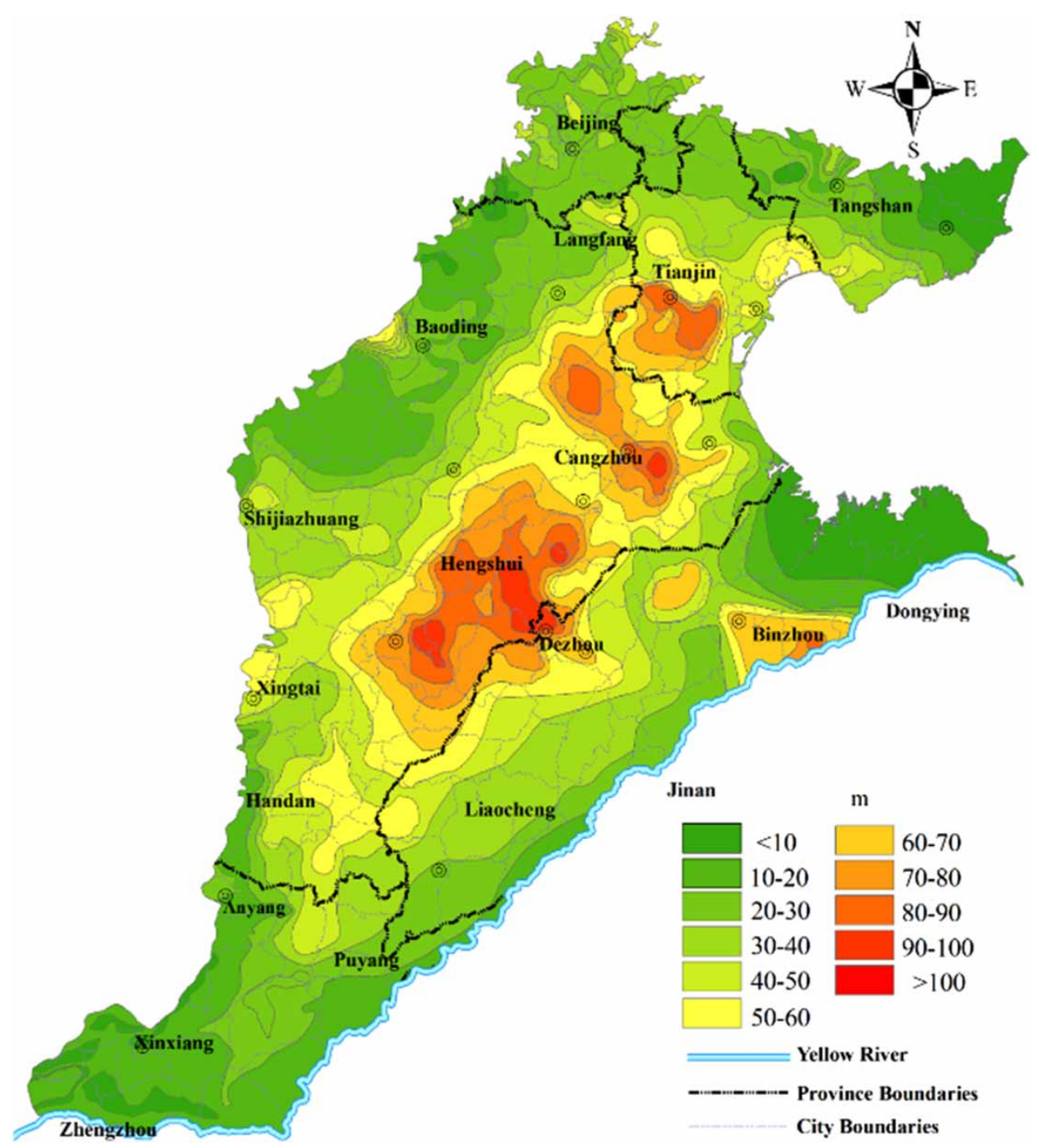

Fig. 4. Spatial distribution of UBW depth in 2005. Source: Institute of Hydrogeology and Environmental Geology (China).

Figure 6(b) shows the results when supplying 5\% of total winter wheat production (TWP). When $P=$ $50 \%$ in the targeting year, the UBW quantity exploited is 0.98 times that of $P=25 \%$. Therefore, the supply of 5\% would be considered as the threshold in the year with $P=50 \%$. When $P=75 \%$ in the targeting year, the UBW quantity exploited is 1.15 times that of $P=25 \%$, which is lower than the threshold. Thus, $5 \%$ of TWP is not the targeting water-saving irrigation.

Figure 6(c) shows the results when there is $10 \%$ of TWP. When $P=75 \%$ in the targeting year, the UBW quantity exploited is 0.92 times that of $P=25 \%$, which is lower than the threshold in the firstcategory to third-category areas. Therefore, 5-10\% of TWP is considered as the proper value in the targeting year with $P=75 \%$. 


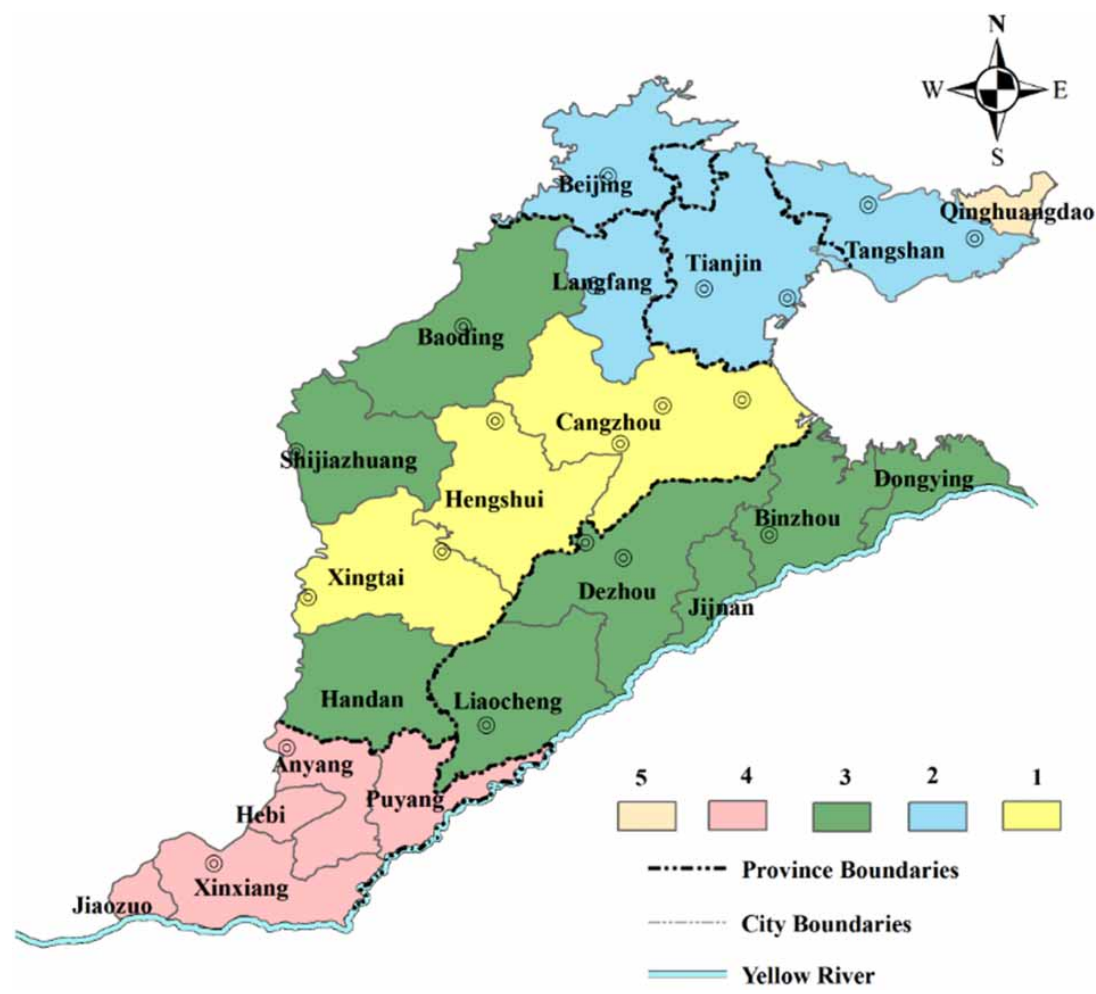

Fig. 5. Comparative superiority of irrigation production efficiency of UBW and its spatial distribution.

\section{Conclusions}

From the above results, we could reach three conclusions:

1. Considering the spatial variability of photosynthetically active radiation, soil organic matter and accumulated temperature, the SVM-GA coupling method could be used to effectively determine water production efficiency of the representative division at every irrigation site in North China Plain.

2. The model for evaluating the comparative advantage of UBW production efficiency was constructed considering the effective precipitation, irrigation efficiency and depth of UBW. The comparative advantage of UBW decreased from north and south to the center but slightly changed from east to west. It was lower in the center than in other areas, and higher in the north than in the south.

3. Combined with different supplies to ensure food security, the way to manage irrigation water in different typical targeting years was obtained. When $P=25 \%$ in the targeting year, there is no need to supply. When $P=50 \%$ in the targeting year, supplying 5\% of TWP is needed and could save $15 \%$ of total UBW. When $P=75 \%$ in the targeting year, supplying $5-10 \%$ of TWP is needed and could save $11-28 \%$ of total UBW. 

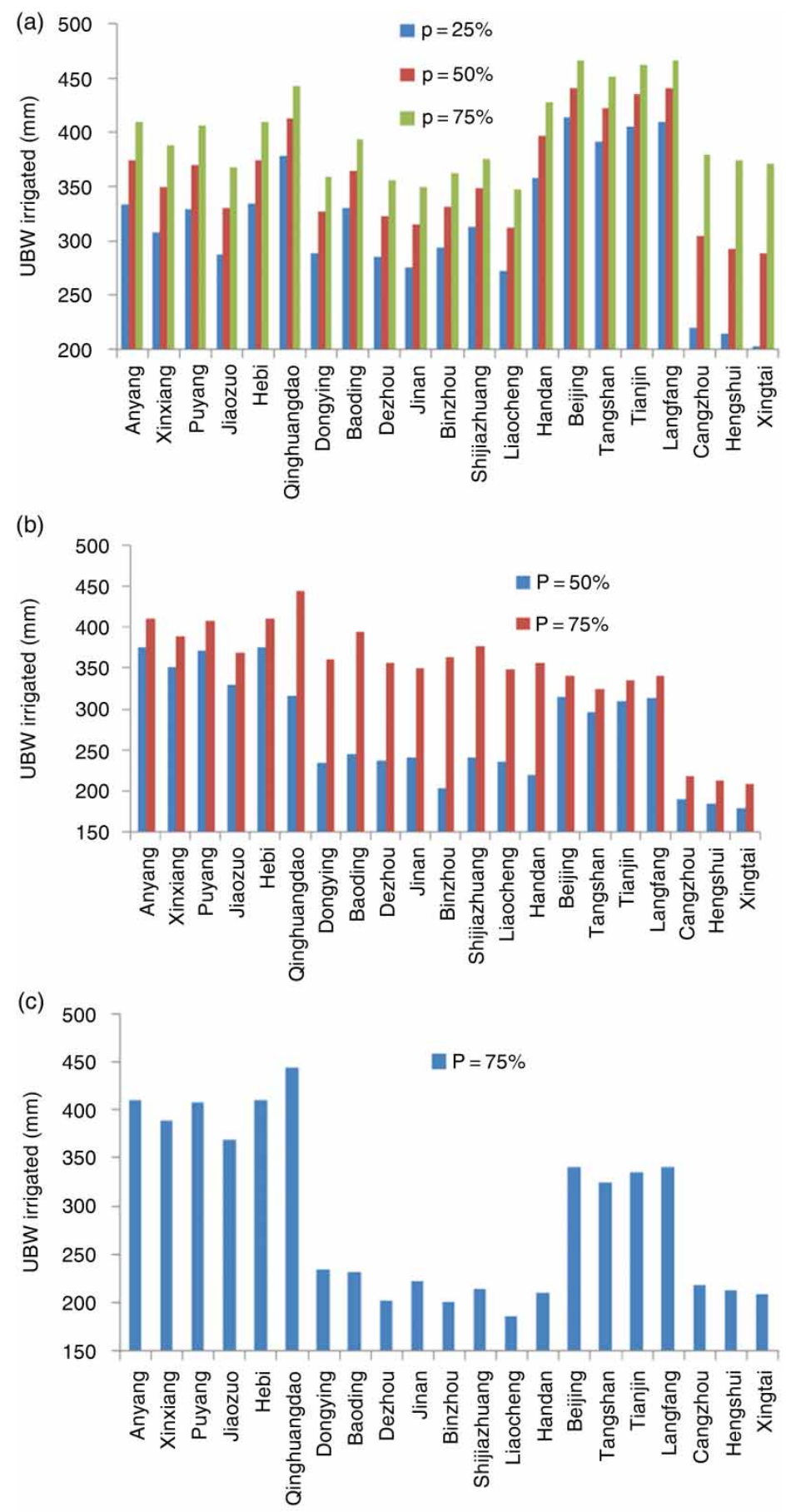

Fig. 6. The UBW quantity irrigated under the situation of different grain reserves. (a) No grain reserve; (b) the grain reserve rate was $5 \%$; (c) the grain reserve rate was $10 \%$. 


\section{References}

Duan, A., Sun, J. \& Liu, Y. (2004). Irrigation Water Quota of Major Crops in North China. China Agricultural Science and Technology Press, Beijing.

Ferrara, V. (2007). Methods and tools for groundwater monitoring aimed at the water resources management under drought conditions. Earth and Environmental Science 62(5), 277-301.

Gong, F. (2003). Optimization of Cropping Pattern and Planting Structure for Water-Saving in North China Plain-Case Study in Shunyi County in Beijing. PhD Thesis, China Agricultural University.

Kipkorir, E. C., Raes, D. \& Massawe, B. (2002). Seasonal water production functions and yield response factors for maize and onion in Perkerra, Kenya. Agricultural Water Management 56(3), 229-240.

Koza, J. R. (1992). Genetic Programming: On the Programming of Computers by Means of Natural Selection. MIT Press, Cambridge.

Li, A. \& Wu, D. (1986). Fuzzy Mathematic Foundations and Its Applications. Xinjiang People Press, Urunqi Shi (in Chinese).

Lin, H. T. \& Lin, C. J. (2003). A Study on Sigmoid Kernels for SVM and the Training of Non-PSD Kernels by SMO-Type Methods. Department of Computer Science and Information on Engineering, Taipei.

Liu, C. (2003). Exploring an ecological benefit of south-to-north water transfers for rehabilitating groundwater systems in The North China Plain. South-to-North Water Transfers and Water Science \& Technology 24(1), 17-19.

Liu, W. Z., Hinsaker, D. J., Li, Y. S. \& Wall, G. W. (2002). Interrelations of yield, evapotranspiration, and water use efficiency from marginal analysis of water production functions. Agricultural Water Management 56(2), 143-151.

Luo, Z., Feng, S. \& Zuo, H. (2006). Preliminary study on water product function for summer corn in Hebei Province. Water Saving Irrigation 1, 17-19.

Machiwal, D., Jha, M. K. \& Mal, B. C. (2011). Assessment of groundwater potential in a semi-arid region of India using remote sensing, GIS and MCDM techniques. Water Resources Management 25(5), 1359-1386.

Mao, Z., Yu, Z. \& Liu, H. (2005). Experimental research on thermal requirement for winter wheat and its leaves. Journal of China Agricultural University 2002(5), 14-19.

Vapnik, V. N. (1995). The Nature of Statistical Learning Theory. Springer Verlag, New York.

Wu, T., Schoenau, J. J., Li, F., Qian, P., Zhang, S., Malhi, S. S. \& Wang, F. (2004). Concepts and relative analytical techniques of soil organic matter. Chinese Journal of Applied Ecology 2004(4), 717-722.

Zeng, Z., Zhao, S. \& Li, Q. (1991). Canopy development, light interception and grain yield in high yielding wheat varieties in Beijing District. Acta Agronomica Sinica 1991(3), 161-170.

Received 14 July 2013; accepted in revised form 26 March 2014. Available online 29 April 2015 